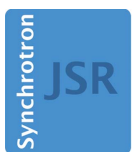

JOURNAL OF SYNCHROTRON RADIATION

ISSN 1600-5775

Received 24 July 2017

Accepted 31 August 2017

Edited by D. Cocco, SLAC National

Accelerator Laboratory, USA

Keywords: free-electron laser; FLASH; SASE; photon pulse duration; photon pulse arrival time; terahertz streaking.

\section{FLASH free-electron laser single-shot temporal diagnostic: terahertz-field-driven streaking}

\author{
Rosen Ivanov, ${ }^{\mathrm{a} *}$ Jia Liu, ${ }^{\mathrm{b}}$ Günter Brenner, ${ }^{\mathrm{a}}$ Maciej Brachmanski ${ }^{\mathrm{a}}$ and \\ Stefan Düsterer ${ }^{\mathrm{a}}$
}

${ }^{\mathbf{a}}$ Deutsches Elektronen Synchrotron - DESY, Notkestrasse 85, 22607 Hamburg, Germany, and ${ }^{\mathbf{b}}$ European XFEL,
Holzkoppel 4, 22869 Schenefeld, Germany. ${ }^{*}$ Correspondence e-mail: rosen.ivanov@desy.de

The commissioning of a terahertz-field-driven streak camera installed at the free-electron laser (FEL) FLASH at DESY in Hamburg, being able to deliver photon pulse duration as well as arrival time information with $\sim 10 \mathrm{fs}$ resolution for each single XUV FEL pulse, is reported. Pulse durations between $300 \mathrm{fs}$ and $<15$ fs have been measured for different FLASH FEL settings. A comparison between the XUV pulse arrival time and the FEL electron bunch arrival time measured at the FLASH linac section exhibits a correlation width of 20 fs r.m.s., thus demonstrating the excellent operation stability of FLASH. In addition, the terahertz-streaking setup was operated simultaneously to an alternative method to determine the FEL pulse duration based on spectral analysis. FLASH pulse duration derived from simple spectral analysis is in good agreement with that from terahertz-streaking measurement.

\section{Introduction}

Since FLASH lases in self-amplified spontaneous emission (SASE) mode each photon pulse is 'unique' and has a different pulse energy, XUV spectrum and pulse duration (Ackermann et al., 2007). Furthermore, due to tiny fluctuations in the electron acceleration the arrival time of the XUV pulses jitters in the order of several tens of femtoseconds. The focus of the online photon diagnostics at FLASH is to measure all fluctuating properties as completely as possible and ideally on a shot-to-shot basis. Due to the burst mode structure of FLASH with up to 800 pulses spaced by $1 \mu$ s (at a repetition rate of $10 \mathrm{~Hz}$ ) such measurements are in general challenging.

Several methods have been developed at FLASH and are in use to determine the pulse energy (Tiedtke et al., 2008), the spectrum (Brenner et al., 2011, 2016) and the arrival time of the electron bunches (Angelovski et al., 2012). However, the XUV pulse duration still lacks an appropriate reliable detector. Several different methods have been pursued (Düsterer et al., 2011) to identify the optimum pulse duration characterization method to cover the temporal range of $10 \mathrm{fs}$ to a few hundred fs (FWHM) within variable wavelength ranging from 4 to $\sim 40 \mathrm{~nm}$, and provide sufficient information on all fluctuating temporal properties. For pump-probe experiments which make use of the ultrashort FEL pulses it is necessary to know the pulse duration for a precise data analysis. Precisely characterized XUV pulse duration is also quite important in all kinds of nonlinear interaction studies where XUV intensity plays a vital role. Moreover, when these experiments advance towards improved temporal resolution, they require more and more accurate measurements of the 
FEL pulse duration. The diagnostics should be non-invasive, thus allowing the experimentalists to maximize the use of the FEL beam for their experimental studies rather than for diagnostics.

The terahertz (THz) streak camera (Grguraš et al., 2012; Frühling et al., 2009) has the potential to deliver single-shot pulse duration information essentially wavelength independent and with a high dynamic range (in pulse duration and pulse energy). Furthermore, it is able to be operated with repetition rates up to several hundred $\mathrm{kHz}$ (potentially even $\mathrm{MHz}$ ). It is also capable of providing the arrival time information between the XUV pulse and the THz driving laser for each single pulse with an accuracy well below $10 \mathrm{fs}$ on a shotto-shot basis. Because of the broad working range (in pulse length, pulse energy, photon energy and repetition rates) the concept can not only be used for FLASH but also for other X-ray FELs (Juranić et al., 2014; Gorgisyan et al., 2017), like, for example, the $\mathrm{MHz}$ repetition-rate European XFEL and LCLS-II.

\section{THz streaking setup}

The THz streak camera uses a noble gas target that is ionized by the FEL pulse (see Fig. 1). The electrons are then subject to the time-varying electric field of the co-propagating $\mathrm{THz}$ field. After interaction with the $\mathrm{THz}$ field, the photoelectrons have changed their momentum component in the direction of the field. If the electron wave packet is short compared with the half-period length of the $\mathrm{THz}$ field, the temporal structure of the wave packet will be mapped onto the kinetic energy distribution of the emitted electrons and depends on the instantaneous $\mathrm{THz}$ vector potential $A(t)$ at the precise

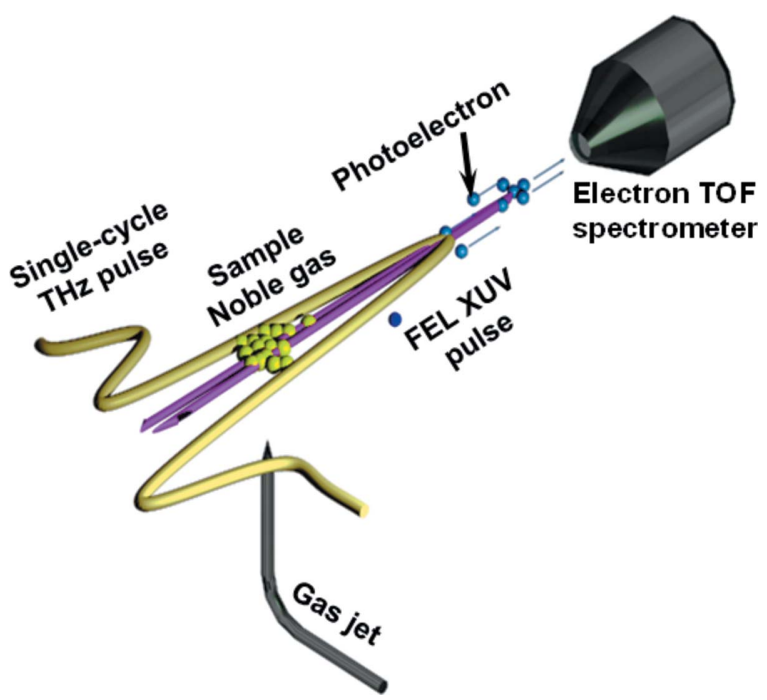

Figure 1

Basic principle of $\mathrm{THz}$ streaking. The FEL pulse ionizes noble gas atoms and the resulting photoelectron kinetic energy distribution is detected by an electron time-of-flight (eTOF) detector. The ionization takes place in the presence of a strong linearly polarized $\mathrm{THz}$ field influencing the kinetic energy distribution of the photoelectrons. Thus, the XUV pulse profile and arrival time are mapped on the kinetic energy distribution by the $\mathrm{THz}$ field. moment of ionization (Itatani et al., 2002; Hentschel et al., 2001). The shift in the kinetic energy relative to the field-free case is $\Delta W\left(t_{\mathrm{o}}\right) \simeq e A\left(t_{\mathrm{o}}\right)\left(2 W_{\mathrm{i}} / m_{\mathrm{e}}\right)^{1 / 2}$, with $W_{\mathrm{i}}$ the initial kinetic energy of the photoelectron, and $e$ and $m_{\mathrm{e}}$ the electron charge and mass. The measured streaked electron energy spectrum reflects the combined temporal and spectral structure of the FEL pulse. The information about the pulse duration, $\tau_{\mathrm{XUV}} \propto$ $\left(\sigma_{\text {streak }}^{2}-\sigma_{\text {ref }}^{2}\right)^{1 / 2}$, can be extracted from the broadening of the peak measured in the photoelectron spectrum due to the presence of the THz field. Here, $\sigma_{\text {streak }}$ relates to the width of the (streaked) photoelectron line in the presence of the $\mathrm{THz}$ radiation while $\sigma_{\text {ref }}$ is that of the 'reference' (without $\mathrm{THz}$ field). The line width also contains information on the FEL bandwidth and the electron time-of-flight (eTOF) detector response. Furthermore, the shift of the streaked photoelectron line provides the arrival time difference between $\mathrm{THz}$ pulses and XUV pulses.

The THz streaking setup was built and installed at FLASH1 at the PG0 branch of the high-resolution plane-grating (PG) monochromator beamline (Martins et al., 2006). FLASH1 is the original FEL line of FLASH, and FLASH2 is a new second FEL line (Faatz et al., 2016). The PG beamline has the capability to use the non-dispersed zero-order beam as well as higher diffraction orders simultaneously. While the zeroth order of the diffraction grating can be guided to the streaking setup at PG0, the dispersed radiation can be simultaneously used in the PG2 beamline branch to, for example, measure the XUV spectrum with high resolution (Gerasimova et al., 2011). By measuring the spectral distribution for each FEL pulse we can disentangle the spectral and the temporal contributions. In addition, the FEL pulse duration can in principle be determined by a second-order correlation analysis of the spectrum as shown by Lutman et al. (2012) and Engel et al. (2016).

The experimental THz streaking setup is depicted in Fig. 2. It consists of a three-axis $(x y z)$ movable support structure, an optical breadboard with optics for the THz generation and

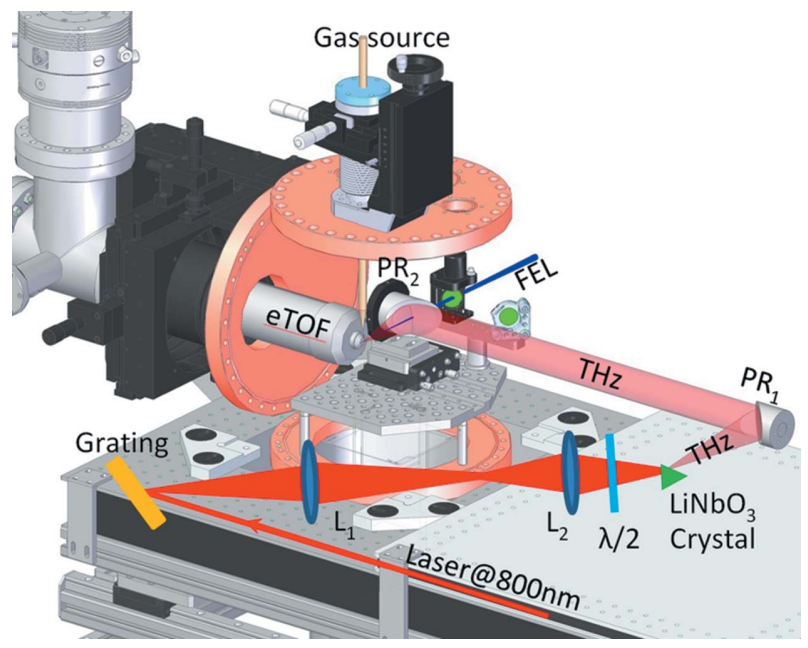

Figure 2

Drawing of the $\mathrm{THz}$ streaking experimental setup with the $\mathrm{THz}$ generation and transport scheme. The chamber, Cube $250 \mathrm{CF}$ and some of the flanges are not shown in order to give a clearer view. 
a compact vacuum UHV chamber (Cube $250 \mathrm{CF}$ ). The THz streaking chamber is equipped with a linear eTOF spectrometer mounted on a three-axis manipulator to allow the optimization of the eTOF position with respect to the interaction point. A $90^{\circ}$ off-axis parabolic mirror (PR2) with focal length of $101.6 \mathrm{~mm}$ is used to collinearly couple in the $\mathrm{THz}$ beam and to focus it to the interaction point. XUV is focused by a $f=500 \mathrm{~mm}$ toroidal mirror through a $3 \mathrm{~mm}$ central hole in the parabola to the noble gas target delivered from a gas needle and spatially overlapped with the focus of the $\mathrm{THz}$ beam. For the FLASH wavelength range, neon proved the ideal target gas due to the high cross section and simple photoelectron line spectrum. The backing pressure in the chamber was around $1 \times 10^{-8} \mathrm{mbar}$ while the pressure in the interaction region was in the low $10^{-7}$ mbar. In this range no space charge effects were observed. Furthermore, the setup houses different diagnostic tools to determine the temporal and spatial overlap between the FEL and $\mathrm{THz}$ pulses. The $\mathrm{THz}$ focus was measured to be $2.1 \mathrm{~mm}$ FWHM (see below, Fig. 4) and is thus significantly larger compared with the XUV beam focused by the toroidal mirror to a spot size of about $300 \mu \mathrm{m}$ FWHM.

\section{THz generation, transport and characterization}

The THz radiation was produced by optical rectification of the FLASH1 pump-probe laser pulses $(10 \mathrm{~Hz}, 800 \mathrm{~nm}, \sim 80 \mathrm{fs}$, $6.5 \mathrm{~mJ})$ using a nonlinear crystal $\left(\mathrm{LiNbO}_{3}\right)$. The process of $\mathrm{THz}$ generation can be viewed as the degenerate case of difference frequency generation for identical frequencies $P(0)=\chi^{(2)}(0, \omega-\omega) E(\omega) E^{*}(\omega)$. The nonlinear polarization $P$ is generated by the incoming field $E$ with frequency $\omega$, mediated by the second-order nonlinear susceptibility $\chi^{(2)}$. If the laser pulse has a duration of less than $1 \mathrm{ps}$, the result will be a single-cycle electromagnetic pulse with frequency contents in the terahertz range. To achieve an efficient $\mathrm{THz}$ generation process, the phase matching was optimized by a tilted pulse front of the driving pump-probe laser pulse as described by Hebling et al. (2002). We used a setup that consists of a diffractive grating (2000 grooves $\mathrm{mm}^{-1}$ ) and $4 f$ two lenses telescope $\mathrm{L}_{1}(f=150 \mathrm{~mm})$ and $\mathrm{L}_{2}(f=75 \mathrm{~mm})$ to image the laser pulse onto the nonlinear crystal, providing the required pulse front tilt. The subsequent $\mathrm{THz}$ beam transport employed two $90^{\circ}$ off-axis gold-coated parabolic mirrors $\mathrm{PR}_{1}(f=$ $152.4 \mathrm{~mm})$ and $\operatorname{PR}_{2}(f=101.6 \mathrm{~mm})$. The transport line was kept as short as possible in air to avoid losses and spectral distortions (Fig. 2). Note that the THz field strength and shape can be directly determined from the streaking spectrogram according to $\Delta W\left(t_{\mathrm{o}}\right) \simeq e A\left(t_{\mathrm{o}}\right)\left(2 W_{\mathrm{i}} / m_{\mathrm{e}}\right)^{1 / 2}$. A THz pulse energy of $15 \mu \mathrm{J}$ and $\mathrm{THz}$ field strength up to $300 \mathrm{kV} \mathrm{cm}-1$ were finally achieved in the interaction region. A single-cycle $\mathrm{THz}$ pulse with a period of about $3 \mathrm{ps}$, centered at $0.6 \mathrm{THz}$, with a vector potential linear slope of at least $500 \mathrm{fs}$ is shown in Fig. 3. The linear part of the $\mathrm{THz}$ field defines the usable time window. The FEL pulse duration including all timing jitters must be in that window, thus the present setup can measure pulse durations of up to $350 \mathrm{fs}$ FWHM. The slope of the $\mathrm{THz}$

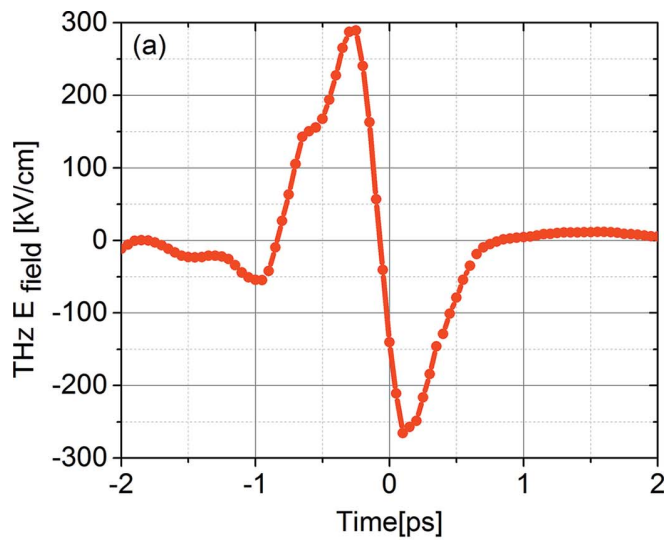

(a)

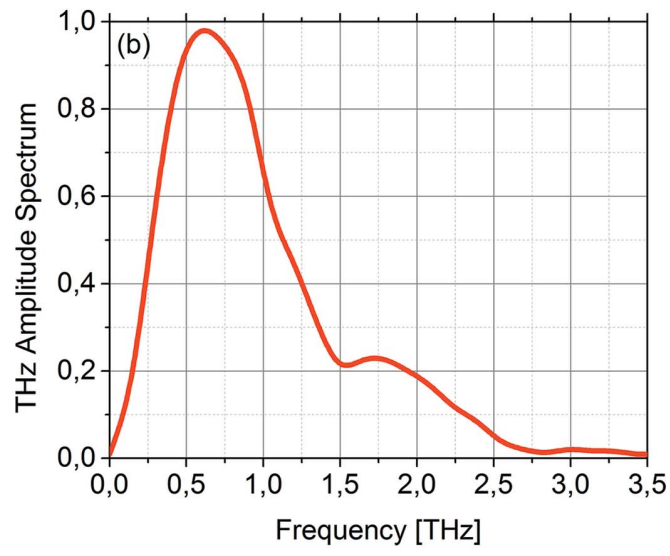

(b)

Figure 3

(a) $\mathrm{THz}$ electric field measured in the interaction region (by $\mathrm{THz}$ streaking) and $(b)$ the resulting $\mathrm{THz}$ spectrum calculated by Fourier transform. The maximum of the spectrum is at $0.6 \mathrm{THz}$.

field, i.e. the so-called streaking speed, $s$, is calculated to be $0.1 \mathrm{eV} \mathrm{fs}^{-1}$. This leads to a streaking resolution of $\sim 10 \mathrm{fs}$ (FWHM) for the current $\mathrm{THz}$ field (Itatani et al., 2002; Frühling et al., 2009).

The propagation of the $\mathrm{THz}$ beam was characterized around the interaction point using a pyroelectric camera (Fig. 4). A round beam profile of $2.1 \mathrm{~mm}$ in FWHM was observed in the focal plane while noticeable astigmatism before and after the focus is observed. Since the phase of the $\mathrm{THz}$ field changes within the Rayleigh range, it is important to understand and quantify the additional broadening of the measured photoelectron line due to the fact that electrons are not only collected from an interaction point but rather from a volume (acceptance volume). Within that volume different electrons see different $\mathrm{THz}$ field strengths. This leads to a phase shift, the so-called Gouy phase shift defined by $\varphi_{\text {Gouy }}(z)=\arctan \left(z / z_{R}\right)$. Thus broadening $\sigma_{\text {Gouy }}=\Delta \varphi / \omega=$ $\Delta \varphi \lambda / 2 \pi c$ because the Gouy phase has to be taken into account in the data analysis: $\tau_{\text {XUV }} \propto\left(\sigma_{\text {streak }}^{2}-\sigma_{\text {ref }}^{2}-\sigma_{\text {Gouy }}^{2}\right)^{1 / 2}$ (Frühling, 2011).

An acceptance 'volume' horizontal length of $\sim 0.5 \mathrm{~mm}$ can be estimated by observing the eTOF signal while moving the eTOF spectrometer. As an example, additional broadenings due to the Gouy phase shift are expected to be 13.3 or $10.2 \mathrm{fs}$ 


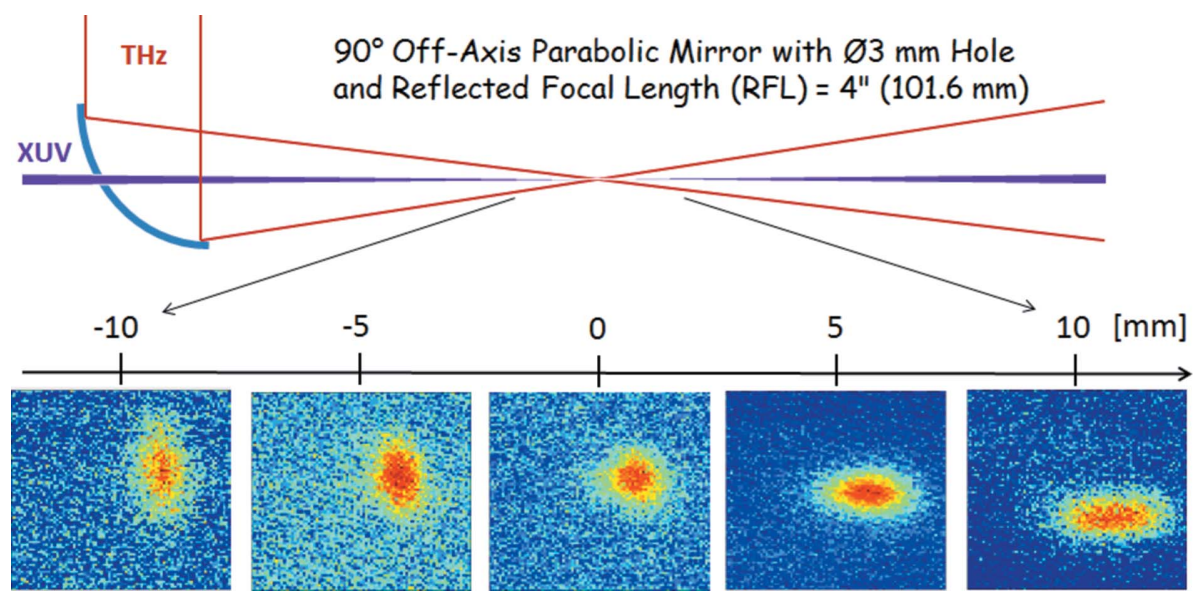

Figure 4

$\mathrm{THz}$ beam shape around the interaction point taken with a pyroelectric camera. In the focal plane the THz spot is $2.1 \mathrm{~mm}$ FWHM.

(FWHM) when measuring directly at the $\mathrm{THz}$ focal plane or $6 \mathrm{~mm}$ from the THz focus. The influence of the Gouy phase becomes significant once it is of the same order as the FEL pulse duration. It turned out that the option to move the eTOF with respect to the $\mathrm{THz}$ focal spot is very useful in order to adapt the $\mathrm{THz}$ setup for different measurement tasks. In addition, the broadening effect can be weakened by minimizing the acceptance volume (e.g. by applying a smaller diameter gas needle). For instance, the above values reduce to 6.3 fs and 4.8 fs (FWHM) if the acceptance volume is $0.25 \mathrm{~mm}$. Another possibility to minimize the Gouy phase induced broadening is to increase the $\mathrm{THz}$ beam size by using a longer focal length parabola at the expense of reducing the $\mathrm{THz}$ field strength which will negatively affect the resolution.

\section{Experimental results}

Up to now several different FEL operation settings have been used to commission the technique over a wide range of pulse durations from $350 \mathrm{fs}$ to less than $15 \mathrm{fs}$ (FWHM). This was achieved by varying the electron bunch charge from 0.44 to less than $0.1 \mathrm{nC}$ for FEL wavelengths of 7, 13 and $20 \mathrm{~nm}$. For each setting the FLASH single-shot pulse duration as well as the arrival time of the XUV pulses with respect to the $\mathrm{THz}$ generating optical (pump-probe) laser were measured. The FLASH1 pump-probe laser is optically synchronized to the FLASH master clock (Schulz et al., 2015). As one example, a recorded time sequence of 5 min (3000 pulses) is shown in Fig. 5; clear inherent fluctuation of the pulse duration around the measured mean value of $\sim 28$ fs (FWHM) is observed.

As a second important result of the commissioning runs we could verify the assumption that the high precision of the electron beam arrival time monitor (BAM) measuring the arrival time of the electron bunches holds also for the arrival time of the XUV pulses in all measured cases. So far this relation has only been demonstrated once for a specific case (Schulz et al., 2015). We determined a very good agreement of the arrival time (with respect to the FEL master timing) of the electrons measured at around $200 \mathrm{~m}$ upstream of the experimental hall in the accelerator tunnel - and the arrival time of the XUV pulses with respect to the pumpprobe laser (producing our $\mathrm{THz}$ ) at the experiment. The shot-to-shot correlation shown in Fig. 6 reveals a correlation width of less than 20 fs r.m.s. for most of the settings investigated so far. This result tells us that one can indeed significantly improve the time resolution of optical-XUV pump-probe experiments by sorting the acquired data according to the electron bunch arrival times measured by the BAM (Savelyev et al., 2017). Moreover, this diagnostic can in future be used to characterize and improve the performance of the FLASH FEL synchronization system and the arrival time detector.

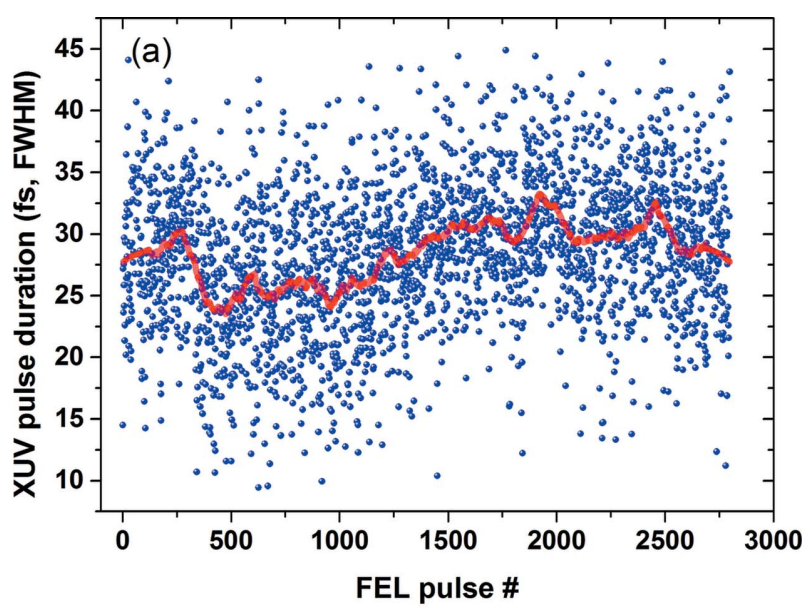

(a)

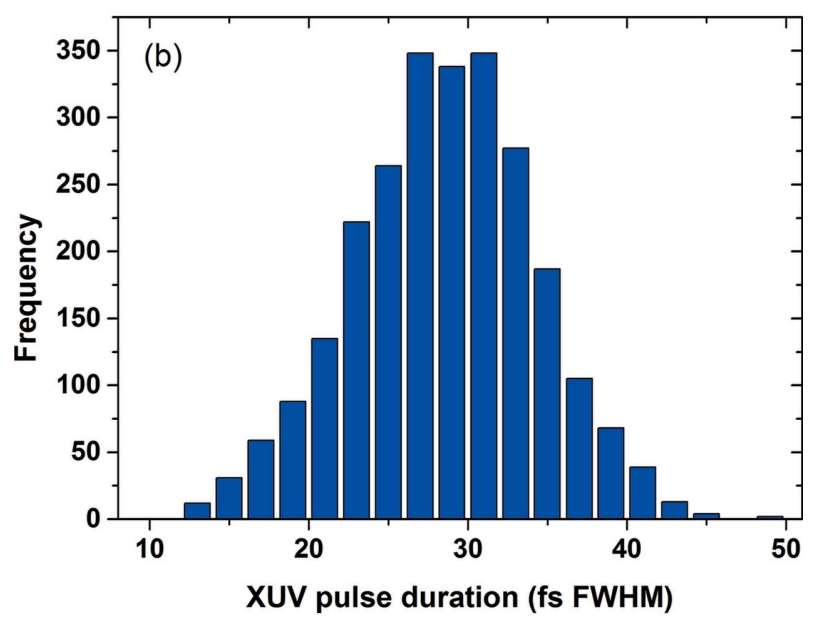

Figure 5

(b)

(a) Single-shot pulse duration for $\sim 3000$ FLASH shots. The red line indicates the mean value ( $\sim 28 \mathrm{fs}$ ) [error bars (not shown) due to the fit uncertainty are of the order of $20 \%]$, and $(b)$ a histogram of the measured pulse durations. 


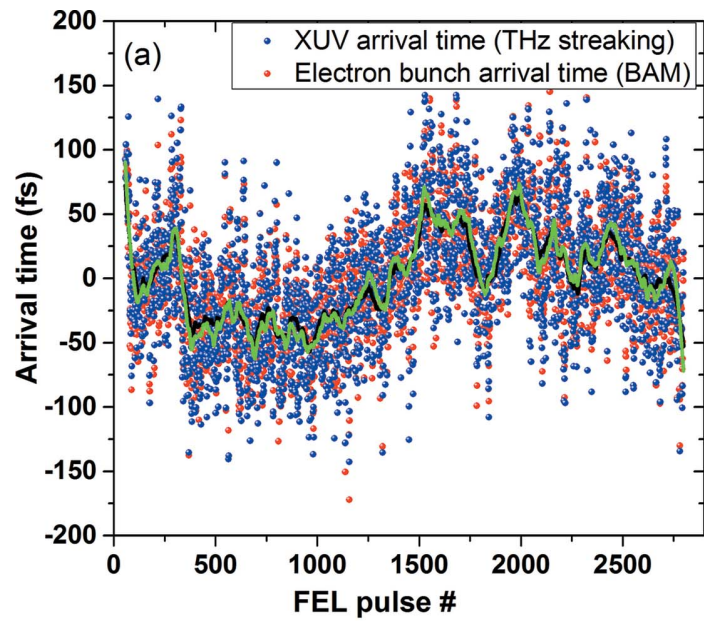

(a)

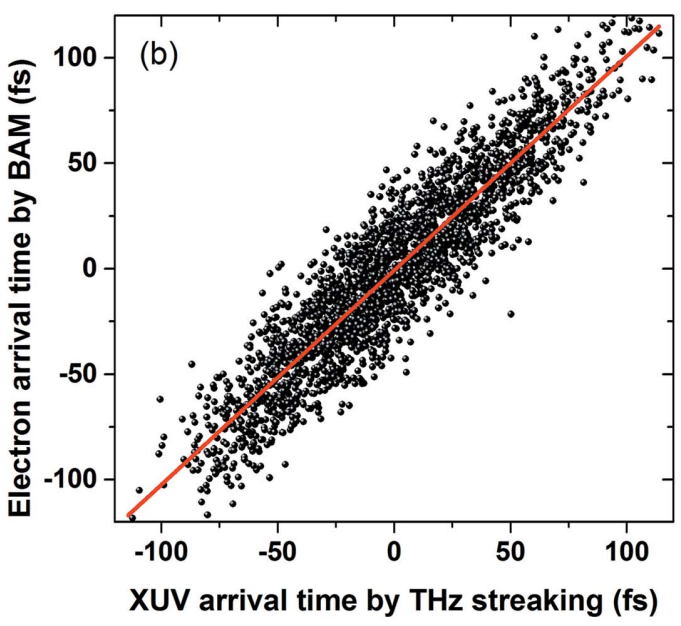

(b)

Figure 6

(a) Arrival time plotted for the same FEL shots as in Fig. 5. The XUV (blue) and electron (red) arrival times agree well on a shot-to-shot basis (dots). Averaging the arrival time over $10 \mathrm{~s}$ (lines, green for $\mathrm{THz}$ streaking data and black for BAM data) still provides a very good agreement. (b) Correlation plot comparing the arrival time measured for electrons (BAM) and the XUV photon pulses at the experiment (streaking) showing a correlation width of only $20 \mathrm{fs}$ r.m.s. The red line indicates the linear fit.

In addition to the single-shot SASE FEL pulse durations and arrival times measured by the $\mathrm{THz}$ streaking setup at PG0, high-resolution XUV spectra can be recorded simultaneously for the exact same FEL pulse. This allows several indirect methods attempting to infer the FEL pulse duration from the spectral distribution to be applied. One rather simple approach is based on counting the spectral spikes which are to a certain extent proportional to the temporal SASE pulse duration (Krinsky \& Gluckstern, 2003). More advanced methods with a presumably larger applicable range are based on one- and two-dimensional second-order spectral correlation $\left[g^{(2)}\right]$ analysis (Engel et al., 2016; Gorobtsov et al., 2017). Fig. 7 shows a first result of the comparison between the pulse duration determined by $\mathrm{THz}$ streaking and the one-dimensional $g^{(2)}$ method for different FEL settings. The analysis for the $g^{(2)}$ approach is explained in detail by Lutman et al. (2012).

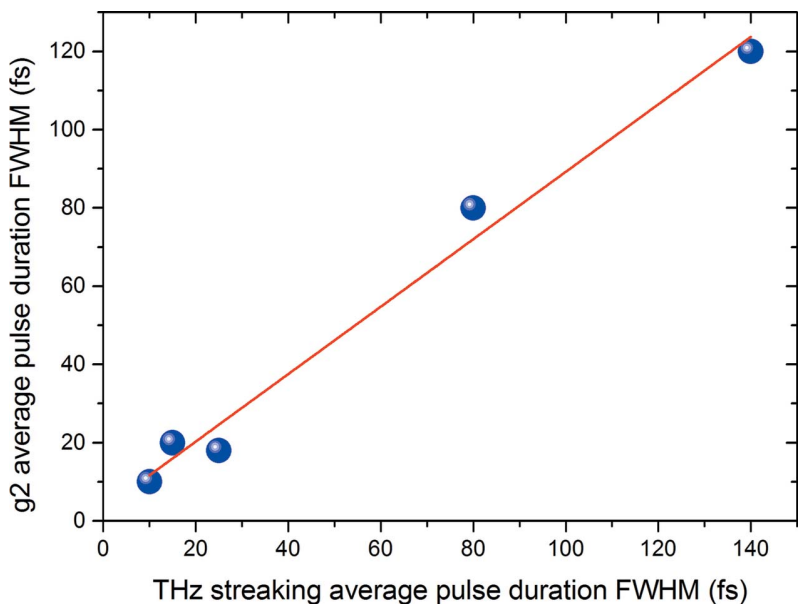

Figure 7

Pulse durations determined by the analysis of the second order $\left[g^{(2)}\right]$ spectral correlation and pulse durations taken from the $\mathrm{THz}$ streaking agree quite well and demonstrate the applicability of the $g^{(2)}$ method over a large range of pulse durations.

The measurements show a good agreement and are promising in that the applicability range of the spectral analysis is indeed rather large and can be used as a simple way to estimate the XUV pulse duration. A publication describing the different approaches and experimental findings in more detail is in preparation

\section{Conclusion}

We report on the installation and commissioning of a pulselength diagnostic setup at FLASH1 based on THz streaking. We have demonstrated single-shot pulse duration measurements of XUV pulses covering the full range of $<15$ fs to $350 \mathrm{fs}$. In addition, we verified the excellent agreement of the electron beam arrival time monitor (BAM) data with the actual arrival time fluctuations of the pump-probe laser at the FLASH1 experimental endstations. A comparison of methods which determine the XUV pulse durations indirectly by spectral analysis shows good agreement with the pulse durations determined by $\mathrm{THz}$ streaking measuring in parallel. The THz streaking technique allows us to measure the single-shot SASE FEL pulse duration in the presence of significant arrival time fluctuations (sometimes even bigger than the FEL pulse duration). One can easily imagine how the analysis of user experiments can be improved if such pulse-resolved online photon diagnostic data are available.

The setup is currently redesigned to fit at FLASH2 into a beamline section in front of all experimental endstations to provide pulse-resolved online photon pulse duration measurements in the future.

\section{Acknowledgements}

The authors acknowledge the work of the scientific and technical team at FLASH and in particular the FLASH laser group. 


\section{References}

Ackermann, W. et al. (2007). Nat. Photon. 1, 336-342.

Angelovski, A., Kuhl, A., Hansli, M., Penirschke, A., Schnepp, S. M., Bousonville, M., Schlarb, H., Bock, M. K., Weiland, T. \& Jakoby, R. (2012). Phys. Rev. ST Accel. Beams, 15, 112803.

Braune, M., Brenner, G., Dziarzhytski, S., Juranić, P., Sorokin, A. \& Tiedtke, K. (2016). J. Synchrotron Rad. 23, 10-20.

Brenner, G., Kapitzki, S., Kuhlmann, M., Ploenjes, E., Noll, T., Siewert, F., Treusch, R., Tiedtke, K., Reininger, R., Roper, M. D., Bowler, M. A., Quinn, F. M. \& Feldhaus, J. (2011). Nucl. Instrum. Methods Phys. Res. A, 635, S99-S103.

Düsterer, S., Radcliffe, P., Bostedt, C., Bozek, J., Cavalieri, A. L., Coffee, R., Costello, J. T., Cubaynes, D., DiMauro, L. F., Ding, Y., Doumy, G., Grüner, F., Helml, W., Schweinberger, W., Kienberger, R., Maier, A. R., Messerschmidt, M., Richardson, V., Roedig, C., Tschentscher, T. \& Meyer, M. (2011). New J. Phys. 13, 093024.

Engel, R., Düsterer, S., Brenner, G. \& Teubner, U. (2016). J. Synchrotron Rad. 23, 118-122.

Faatz, B. et al. (2016). New J. Phys. 18, 062002.

Frühling, U. (2011). J. Phys. B, 44, 243001.

Frühling, U., Wieland, M., Gensch, M., Gebert, Th., Schütte, B., Krikunova, M., Kalms, R., Budzyn, F., Grimm, O., Rossbach, J., Plönjes, E. \& Drescher, M. (2009). Nat. Photon. 3, 523-528.

Gerasimova, N., Dziarzhytski, S. \& Feldhaus, J. (2011). J. Mod. Opt. 58, 1480-1485.

Gorgisyan, I., Ischebeck, R., Erny, C., Dax, A., Patthey, L., Pradervand, Cl., Sala, L., Milne, C., Lemke, H. T., Hauri, C. P., Katayama, T., Owada, S., Yabashi, M., Togashi, T., Abela, R., Rivkin, L. \& Juranić, P. (2017). Opt. Express, 25, 2080.

Gorobtsov, O. Yu., Mercurio, G., Brenner, G., Lorenz, U., Gerasimova, N., Kurta, R. P., Hieke, F., Skopintsev, P., Zaluzhnyy, I., Lazarev, S., Dzhigaev, D., Rose, M., Singer, A., Wurth, W. \& Vartanyants, I. A. (2017). Phys. Rev. A, 95, 023843.

Grguraš, I., Maier, A. R., Behrens, C., Mazza, T., Kelly, T. J., Radcliffe, P., Düsterer, S., Kazansky, A. K., Kabachnik, N. M., Tschentscher, Th., Costello, J. T., Meyer, M., Hoffmann, M. C., Schlarb, H. \& Cavalieri, A. L. (2012). Nat. Photon. 6, 852-857.
Hebling, J., Almási, G., Kozma, I. Z. \& Kuhl, J. (2002). Opt. Express, 10, 1161-1166.

Hentschel, M., Kienberger, R., Spielmann, Ch., Reider, G. A., Milosevic, N., Brabec, T., Corkum, P. U., Heinzmann, U., Drescher, M. \& Krausz, F. (2001). Nature (London), 414, 509-513.

Itatani, J., Quéré, F., Yudin, G. L., Ivanov, M. Yu., Krausz, F. \& Corkum, P. B. (2002). Phys. Rev. Lett. 88, 173903.

Juranić, P. N., Stepanov, A., Ischebeck, R., Schlott, V., Pradervand, C., Patthey, L., Radović, M., Gorgisyan, I., Rivkin, L., Hauri, C. P., Monoszlai, B., Ivanov, R., Peier, P., Liu, J., Togashi, T., Owada, S., Ogawa, K., Katayama, T., Yabashi, M. \& Abela, R. (2014). Opt. Express, 22, 30004-30012.

Krinsky, S. \& Gluckstern, R. L. (2003). Phys. Rev. ST Accel. Beams, 6 , 050701.

Lutman, A. A., Ding, Y., Feng, Y., Huang, Z., Messerschmidt, M., Wu, J. \& Krzywinski, J. (2012). Phys. Rev. ST Accel. Beams, 15, 030705 .

Martins, M., Wellhöfer, M., Hoeft, J. T., Wurth, W., Feldhaus, J. \& Follath, R. (2006). Rev. Sci. Instrum. 77, 115108.

Savelyev, E., Boll, R., Bomme, C., Schirmel, N., Redlin, H., Erk, B., Düsterer, S., Müller, E., Höppner, H., Toleikis, S., Müller, J., Kristin Czwalinna, M., Treusch, R., Kierspel, Th., Mullins, T., Trippel, S., Wiese, J., Küpper, J., Brauße, F., Krecinic, F., Rouzée, A., Rudawski, P., Johnsson, P., Amini, K., Lauer, A., Burt, M., Brouard, M., Christensen, L., Thøgersen, J., Stapelfeldt, H., Berrah, N., Müller, M., Ulmer, A., Techert, S., Rudenko, A. \& Rolles, D. (2017). New J. Phys. 19, 043009.

Schulz, S., Grguraš, I., Behrens, C., Bromberger, H., Costello, J. T., Czwalinna, M. K., Felber, M., Hoffmann, M. C., Ilchen, M., Liu, H. Y., Mazza, T., Meyer, M., Pfeiffer, S., Prędki, P., Schefer, S., Schmidt, C., Wegner, U., Schlarb, H. \& Cavalieri, A. L. (2015). Nat. Commun. 6, 5938.

Tiedtke, K., Feldhaus, J., Hahn, U., Jastrow, U., Nunez, T., Tschentscher, T., Bobashev, S. V., Sorokin, A. A., Hastings, J. B., Möller, S., Cibik, L., Gottwald, A., Hoehl, A., Kroth, U., Krumrey, M., Schöppe, H., Ulm, G. \& Richter, M. (2008). J. Appl. Phys. 103, 094511. 